\title{
Effects of context in judgments of sweetness and pleasantness
}

\author{
DWIGHT R. RISKEY \\ Monell Chemical Senses Center, Philadelphia, Pennsylvania 19104 \\ ALLEN PARDUCCI \\ University of California, Los Angeles, Los Angeles, California 90024 \\ and \\ GARY K. BEAUCHAMP \\ Monell Chemical Senses Center, Philadelphia, Pennsylvania 19104
}

\begin{abstract}
Dramatic effects of the immediate stimulus context were demonstrated for ratings of sweetness and also for ratings of pleasantness of soft drinks containing different concentrations of sucrose. The same drinks were rated sweeter when the lower concentrations were presented more frequently, less sweet when the higher concentrations were presented more frequently. A quasi-normal distribution of frequencies yielded ratings falling between the two skewed distributions. Ratings of sweetness were accurately predicted by Parducci's (1974) range-frequency model of judgment, which was originally developed to explain contextual effects in other psychophysical dimensions. Ratings of pleasantness were also affected by context; the highest ratings were assigned to concentrations of intermediate sweetness in their respective contexts.
\end{abstract}

Judgments of size, weight, loudness, and brightness clearly depend upon the frame of reference, or context, against which those judgments are made (Helson, 1964; Parducci, 1963, 1965, 1974; Poulton, 1968). Stevens' (1958) example of "a large mouse that ran up the trunk of a small elephant" is absurd in an absolute sense, but reasonable if it is understood that judgments are relative to context. In this case, the two different species define two separate contexts.

Although the relativism of the judgments is widely considered a universal phenomenon, judgments of taste may not be sensitive to effects of context. Several studies have reported that although the perceived sweetness of foods increases with increasing concentration of sucrose, ratings of pleasantness of tastes typically increase and then decrease with increasing sucrose concentration (Kocher \& Fisher, 1969; Moskowitz, 1971; Moskowitz, Kluter, Westerling, \& Jacobs, 1974; Moskowitz, Kumaraiah, Sharma,

Thanks are extended to Robert H. Cagan, Carol M. Christensen, and Charles J. Wysocki for their valuable criticisms and suggestions on earlier versions of the manuscript and to Barry Fabius for technical assistance. The first author was partially supported by NIH Training Grant 5-T32-NS-07068-03. Requests for reprints should be addressed to Dwight R. Riskey, Monell Chemical Senses Center, 3500 Market Street, Philadelphia, Pennsylvania 19104.
Jacobs, \& Sharma, 1975). Moskowitz et al. (1974) found that the sucrose concentration that produces the peak in the pleasantness function varies depending upon the food tasted but that its relationship to judged sweetness is invariant. The breakpoint in pleasantness thus occurred, not at a fixed concentration of sucrose, but at a fixed rating of sweetness. According to Moskowitz et al. (1974), the data suggested that the pleasantness response is directly linked to sensory sweetness. Furthermore, Moskowitz et al. concluded that, since different foods (and different experiments) produce the same relationship between judged sweetness and pleasantness, sweetness must be an absolute rather than a relative perception. That is, judgments of sweetness are context-free; judgments of pleasantness follow directly from judgments of sweetness.

If sweet taste is, in fact, free of contextual effects, it must be fundamentally different from other sensory responses. It seems more likely that judgments of sweet substances only appear context-free in existing experiments because context was not effectively manipulated. Although several foods were tested by Moskowitz et al. (1974), each food was tested at an equal number of high and low levels of sucrose. Because different foods may define different judgment domains (just as in the earlier example different species define different contexts for size judgments), similar frequency distributions of sucrose concentra- 
tions for each food might be expected to produce similar judgments.

The invariant relationship observed between sweetness and the pleasantness breakpoint might also be explained by the practice of requiring both sweetness and pleasantness judgments of each stimulus. Although Moskowitz (1977a) has argued that simultaneous judgments are advantageous because they emphasize to subjects the differences between sweetness and pleasantness, it is also possible that the two types of judgments become artificially linked in the process. Simultaneous judgments may force subjects to think of pleasantness in terms of sweeiness and sweetness in terms of pleasantness. Variations in the context of sweetness, therefore, may be inappropriately reflected by changes in ratings of both pleasantness and sweetness although the two dimensions may normally be independent.

The present experiment tested the effects of context on pleasantness and sweetness by varying the relative frequencies of sweet and unsweet beverages during the experimental session. If perceived sweetness is not influenced by context, these manipulations should have no effect on judged sweetness or pleasantness. However, if context affects taste perception, and if it differentially affects sweetness and pleasantness judgments, then the relationship between sweetness and the breakpoint in pleasantness should change. In order to evaluate whether making both sweetness and pleasantness judgments of each stimulus influences judgments, different types of reporting conditions were used. In one condition, subjects were requested to rate pleasantness and sweetness; the other conditions required judgments of either pleasantness or sweetness, but not both.

\section{METHOD}

Subjects were presented a series of cherry-flavored soft drinks varying in sucrose congentration, and were instructed to judge sweelness, pleasantness, or both for each stimulus.

\section{Stimuli}

Beverages containing seven concentrations of reagent grade sucrose (Fisher Scientific Company) were prepared ranging from .06 to $1.80 \mathrm{M}$ in equal $\log$ steps. Each beverage contained an equal amount of cherry flavoring.' The stimuli were presented in plastic soufflé cups, each containing approximately $10 \mathrm{ml}$ of of beverage. The beverages were served at room temperature (approximately $21^{\circ} \mathrm{C}$ ) but were refrigerated during all storage periods. The beverages were prepared at least $48 \mathrm{~h}$ before testing but never used after more than 8 days' storage.

\section{Procedure and Design}

Subjects were randomly assigned to conditions requiring judgments of (a) sweetness only or pleasantness only, or (b) both sweetness and pleasantness for each beverage. In the singlejudgment conditions, the subjects were randomly divided so that half made judgments of pleasantness only and half made judgments of sweetness only. For the condition in which both sweetness and pleasantness were judged, half of the subjects made sweetness judgments first, half made pleasantness judgments first. Therefore, three of these four reporting conditions yielded sweetness judgments; three yielded pleasantness judgments.
Table 1

Frequency of Presentation (per Block of 21 Trials) of Each of Seven Concentrations of Sucrose in the Three Contexts

\begin{tabular}{cccccccc}
\hline & \multicolumn{7}{c}{ Sucrose Molarity } \\
\cline { 2 - 8 } Context & .06 & .11 & .19 & .34 & .59 & 1.0 & 1.8 \\
\hline Positive Skew & 7 & 4 & 3 & 3 & 2 & 1 & 1 \\
Quasi-Normal & 1 & 2 & 4 & 7 & 4 & 2 & 1 \\
Negative Skew & 1 & 1 & 2 & 3 & 3 & 4 & 7 \\
\hline
\end{tabular}

Within each of the reporting conditions, the beverages were presented in one of three stimulus distributions (contexts) in which the rejative frequencies of the different concenirations were varied. In the negatively skewed distribution, beverages of high sucrose concentration were most frequent. In the positively skewed context, beverages of low sucrose concentration were most frequent. A quasi-normal distribution was also presented in which beverages of extremely high and extremely low concentrations of sucrose were relatively rare. The frequencies of stimuli in each context are presented in Table 1.

Each subject judged two complete replicates of the 21 presentátions comprising a context. Within each replicate, concentrations were presented in random order. At the beginning of the experimental session, 10 practice trials, representative of the context, were presented to acquaint subjects with the rating scale and introduce the context. In the positively skewed context, for example, the lowest beverage was presented on three trials, the highest beverage was presenteo oniy once. The practice trials therefore approximalely reflected the context to be presented and established a frame of reference for judgments to follow. The first 10 trials were not identified as practice to subjects.

Ratings were made in terms of 9-point category scales ranging from 9 (extremely sweet or extremely pleasant) to 1 (no sweetness whatsoever or extremely unpleasant) for sweetness. and pleasantness scales, respectively. A response of 5 on the pleasantness scale represented hedonic neutrality. The numerical scales with verbal category labels were posted for reference throughout the tasting session.

A standard sip-and-spit procedure was employed in which subjects sipped all of the beverage contained in the cup, expectorated it, verbally reported judged sweetness and/or pleasantness, and finally rinsed thoroughly with deionized water in preparation for the next beverage. Trials were seif-paced aithough nost subjects finished the 52 -beverage taste session within 40 to $50 \mathrm{~min}$.

\section{Subjects}

The subjects were 48 student volunteers from the University of Pennsylvania and Drexel University. Each was randomly assigned to one of two basic reporting types and one of the three contexts. Thus, 24 subjects judged either pleasantness or sweetness (12 subjects judging each); 24 subjects judged both. Each subject was tested individually and was paid $\$ 4$ for participating.

\section{RESULTS}

\section{Sweetness}

Figure IA shows that rated sweetness increases monotonically with increasing concentration of sucrose but that there are dramatic effects of context. A given concentration is judged much sweeter in the positively skewed distribution, in which beverages of lower concentration predominated, than in the negatively skewed distribution, in which beverages of higher concentration predominated. The quasi-normal context produced the sigmoidal function characteristic of such contexts (Parducci \& Perrett, 1971). Judgments 


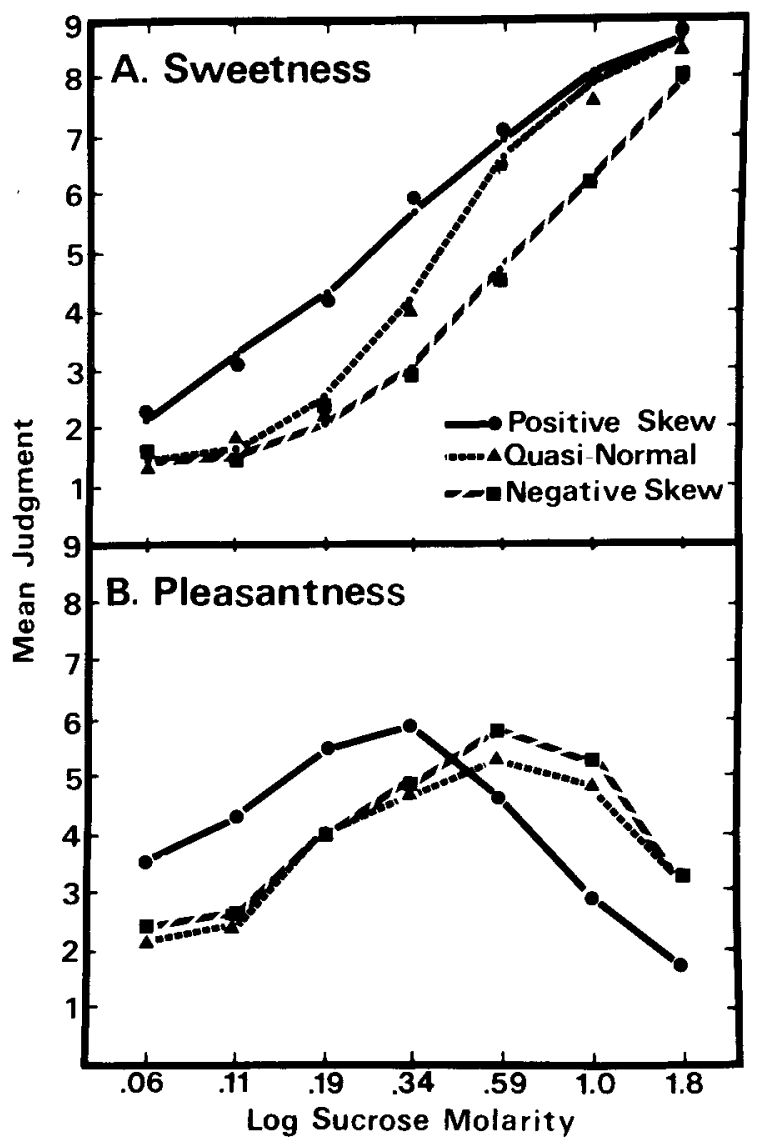

Figure 1. Mean ratings of sweetness and pleasantness for seven concentrations of sucrose presented in three different frequency distributions. Each panel includes the judgments of 36 subjects (12 of the original 48 subjects judged sweetness or pleasantness only), the responses of 12 subjects making up each curve. In Panel $A$, the large points represent empirical mean ratings; the connected points represent the fit of the range-frequency model to the data. All points in Panel B represent empirical mean ratings.

of this symmetrical function fell between the two skewed distributions. Analysis of variance showed significant main effects of both context and concentration $[F(2,27)=34.04$ and $F(6,162)=632.76$, $\mathrm{p}<.01$, respectively]. The interaction between context and concentration also was significant $[F(12,162)=$ $9.84, \mathrm{p}<.01]$.

\section{Pleasantness}

The general shape of the curves for pleasantness (Figure 1B) is consistent with the findings of Moskowitz (1971) and others. The peaks of the curves (the breakpoints) all fall within the .30-to .60-M range previously reported for simple sucrose solutions (Chappell, 1953; Moskowitz, 1971) and sucrose in a variety of foods and beverages (Moskowitz, 1977b; Moskowitz et al., 1974). The effect of sucrose concentration across contexts was significant $[F(6,162)$ $=24.48, \mathrm{p}<.01]$.
Although the average pleasantness of the three contexts did not differ $[F(2,27)=.36]$, the interaction between context and concentration was significant $[F(12,162)=6.28, p<.01]$. Lower concentrations were judged more pleasant in the positive context than in the symmetrical or negative contexts. Higher concentrations were judged less pleasant in the positive context.

\section{Breakpoints}

The effect of context on the pleasantness functions is substantiated by a further analysis that considers only the breakpoints demonstrated for each subject. Because each subject rated two replicates of the 21 beverages, two estimates of each subject's breakpoint in pleasantness were obtained. In a few cases, adjacent concentrations in the series were both judged maximally pleasant. The concentration midway between was then used as the breakpoint. Mean breakpoints for each context are presented in the top row of Table 2 . The maximally pleasant beverage in the positive context contained approximately half as much sucrose as the breakpoints in the other two contexts. Analysis of variance of these breakpoints showed a significant main effect of context $[F(2,27)$ $=7.24, \mathrm{p}<.01]$.

To determine whether breakpoints for each context occurred at a single level of subjective sweetness, breakpoints were also calculated in terms of the rated sweetness, rather than physical concentration, of the maximally pleasant beverage for each subject. Thus, the concentration of sucrose corresponding to each breakpoint for pleasantness was calculated; the subject's rating of sweetness for that concentration was then determined. These values, shown in the bottom row of Table 2 , differ significantly with context $[\mathrm{F}(2,18)=6.96, \mathrm{p}<.01]$. Although the average breakpoint was higher in sucrose concentration for the negative context, it was still judged lower in sweetness than the breakpoints for positive or symmetrical contexts. Conversely, the positive context produced lower breakpoints in terms of concentration, but higher breakpoints in terms of ratings.

Table 2

Mean Breakpoints in Pleasantness Calculated According to Sucrose Concentration and Judged Sweetness

\begin{tabular}{|c|c|c|c|c|c|c|}
\hline \multirow{3}{*}{$\begin{array}{l}\text { Measure of } \\
\text { Breakpoint }\end{array}$} & \multicolumn{6}{|c|}{ Context } \\
\hline & \multicolumn{2}{|c|}{ Positive } & \multicolumn{2}{|c|}{ Quasi-Normal } & \multicolumn{2}{|c|}{ Negative } \\
\hline & Mean & SD & Mean & SD & Mean & $\mathrm{SD}$ \\
\hline $\begin{array}{l}\text { Sucrose Concentration } \\
\text { Rated Sweetness }\end{array}$ & $\begin{array}{r}.28 \\
5.48\end{array}$ & $\begin{array}{l}.08 \\
.91\end{array}$ & $\begin{array}{r}.53 \\
6.56\end{array}$ & $\begin{array}{r}.11 \\
1.98\end{array}$ & $\begin{array}{r}.57 \\
4.20\end{array}$ & $\begin{array}{r}.10 \\
1.15\end{array}$ \\
\hline
\end{tabular}

Note-Sucrose concentration was based on pleasantness ratings of all 12 subjects in each context. Rated sweetness was based on ratings of the eight subjects in each context who made both pleasantness and sweetness judgments (see text for method of calculation). 
The breakpoints for the symmetrical distribution may be spuriously high. Because the slope of the sweetness function around the three middle sucrose concentrations is extremely steep, only a slight change in the breakpoint for concentration would produce a large change in judgment breakpoint. Specifically, a one-third log-step drop in the breakpoint for concentration would yield a breakpoint for judgment approximately midway between the breakpoints for the two skewed contexts. The high variability in judgment breakpoints for the symmetrical distribution (Table 2) lends credence to the hypothesis that the high judgment breakpoint for the symmetrical skew might be inaccurate.

\section{Sensory Adaptation}

It could be argued that sensory adaptation produces the obtained contextual effects. For example, in the negative context, fatigue or adaptation of taste receptors may be more acute (since beverages with high sucrose concentration are more frequent) than in the positive context. Most evidence suggests, however, that fatigue and sensory adaptation to repeated presentations of sweet beverages have small effects on sensitivity (cf. Amerine, Pangborn, \& Roessler, $1965)$ and that these effects are further reduced by rinsing between tastes (O'Mahony \& Dunn, 1974).

In addition, if sensory adaptation is responsible for contextual effects, adaptation should be strongly evident in the effects of one stimulus on the next. For example, a given stimulus should be judged less sweet when following a beverage higher in sucrose than when following a beverage lower in sucrose. As a test, those beverages immediately preceded by a higher concentration on one trial but by a lower concentration on another trial were compared for each subject. An estimate of the effect of sequence for each subject was thereby obtained. Although beverages preceded by a higher sucrose concentration were judged significantly less sweet than the same beverages preceded by a lower concentration $[F(1,33)=17.79, p<.01]$, the effect was small, averaging less than one-half of a judgment category. This finding is consistent with reports from other perceptual domains that effects of presentation sequence are small relative to the larger effects of overall stimulus distribution (cf. Ward \& Lockhead, 1971).

\section{Replicates}

Despite receiving a large number of stimuli in a relatively short tasting period, subjects did not show evidence of reporting fatigue. A comparison of the first replicate of 21 beverages to the second replicate yielded nonsignificant main effects for both sweetness and pleasantness $[F(1,27)=4.15$ and .01 , respectively]. Moreover, none of the two-way or three- way interactions associated with replicates was significant in either analysis.

\section{Reporting Types}

The effect of type of reporting was analyzed separately for pleasantness and sweetness ratings. In the initial analyses, three reporting types were used: (a) sweetness followed by pleasantness, (b) pleasantness followed by sweetness, and (c) sweetness only or pleasantness only. Neither sweetness nor pleasantness analyses showed main effects of type of reporting $[\mathrm{F}(2,27)=.50$ and .56 , respectively $]$. The two-way interactions associated with type of reporting (i.e., Context by Reporting Type and Concentration by Reporting Type) were also nonsignificant for both analyses.

A separate analysis included only subjects who had rated both the sweetness and pleasantness of each stimulus. Again, neither sweetness nor pleasantness ratings were affected by order of reporting $[F(1,18)$ $=1.22$ and .43 , respectively]. Interactions between concentration, order of reporting, and context were also not significant. In addition, effects of order of reporting were examined for the highest concentration of sucrose, where there is greatest discrepancy between judgments of sweetness and pleasantness. Here, also, reporting order had no effect on judgments of either sweetness or pleasantness $[F(1,18)$ $=.73$ and .42 , respectively]. It thus appears that ratings of either pleasantness or sweetness were not affected by whether the task required ratings on the other dimension or on the order in which those judgments were reported.

\section{DISCUSSION}

Effects of context are evident in judgments of both sweetness and pleasantness. This suggests that judgments of taste, like judgments of other psychophysical stimuli, are relative, not absolute, in nature. When stimuli high in sucrose concentration are most frequent, mean judgments of the sweetness of a given beverage are low. The pleasantness breakpoint is higher, in this case indicating that it takes a greater molarity of sucrose to produce maximum pleasantness when there are more sweet stimuli in the context.

The hypothesis that the concentration judged maximally pleasant is directly linked to a given rating of sweetness (Moskowitz et al., 1974) is only partly supported by the data. The rated sweetness of the pleasantness breakpoints in the three contexts differ significantly. However, had the breakpoints not changed with context, the subjective sweetness of the common breakpoint would have differed by a much greater margin. For example, suppose the positive and negative skews shared a breakpoint at the middle 
of the distribution of concentrations. This breakpoint would be rated approximately 3.0 in sweetness for the negative context but approximately 5.9 in the positive context. This difference of almost 3 rating categories is much greater than the observed difference of approximately 1.3 categories (Table 2). The observed shifts in the breakpoint with context therefore tended to minimize the differences between the subjective sweetness of those breakpoints.

\section{Judgment Theory}

The observed relativism for ratings of sweetness is strikingly consistent with the range-frequency theory developed to explain contextual effects in research using other psychophysical dimensions (Parducci, 1974). The predicted values shown in Figure 1A were derived from the basic range-frequency equation:

$$
C_{i j}=w R_{i}+(1-w) F_{i j},
$$

in which $C_{i j}$, the category rating of the $i^{\text {th }}$ stimulus in the $j^{\text {th }}$ context, is a weighted mean of $R_{i}$, its inferred position in the subjectve range of contextual stimuli, and $F_{i j}$, its percentile rank in the $j$ th context (linearly transformed to the $1-9$ scale). Since $F_{i j}$ is calculated a priori from the stimulus frequencies, and since $R_{i}$ is assumed to be independent of skewing for contexts which, as in the present case, share the same endpoint, the differences between the three functions in Figure 1A depend on only the single weighting parameter, $w$. The best-fit value of $w$ for the obtained ratings of sweetness is approximately .4. In terms of the theory, this value means that the tendency to put the same number of stimulus presentations in each category (the frequency principle) is stronger than the tendency to rate each stimulus in accordance with its position in the stimulus range (the range principle). This small value for $\mathrm{w}$, compared with the usual $.5, .6$, or higher, indicates that the effects of skewing can be as great or even greater for ratings of sweetness than for the other dimensions to which the model has been applied, such as visual size (Parducci \& Perrett, 1971) or numerical magnitude (Parducci, Calfee, Marshall, \& Davidson, 1960). The excellent fit of the model suggests that ratings of sweetness follow the same principles of relativistic judgment as do ratings of other psychophysical dimensions.

The ratings of pleasantness are more difficult to analyze, because their relationship to the concentrations of sucrose is nonmonotonic and also because the present contexts for pleasantness seem to include tastes from outside the experimental set. For each of the present contexts, the highest rating of pleasantness is assigned to an intermediate concentration of sucrose, with fairly symmetrical gradients as a func- tion of distance (either up or down) from this most pleasant concentration. Presumably, the same rangefrequency principles would govern these pleasantness ratings (as appears to be the case for odors, cf. Sandusky \& Parducci, 1965), but this cannot be determined from the present data. Since the highest mean ratings for any of the contexts scarcely rise above the middle of the scale (within one step of "neutral" pleasantness), one can assume that these contexts include other tastes more pleasant than any of those present in this experiment. What is surprising is that the concentrations rated most pleasant are so close to the concentrations rated at the middle of the respective scales of sweetness.

\section{Context for Individuals}

Several investigators have reported significant numbers of subjects who fail to demonstrate a breakpoint or asymptote in the pleasantness function for sucrose (e.g., Moskowitz, 1977a, 1977b; Pangborn, 1970). For many of these subjects, judgments of pleasantness closely parallel judgments of sweetness. For others, judgments may actually decline monotonically with increasing concentration of sucrose. The present research, however, failed to detect a single subject who did not show a breakpoint at or before the 1-M sucrose concentration on at least one of the 21 -trial replicates. It is unlikely that subjects in previous studies misinterpreted the task, judging sweetness rather than pleasantness. These studies range widely in the scaling procedure used and the type of instructions presented. Furthermore, the lack of differences between types of reporting in the present case deemphasizes the importance of these differences in procedure. Instead, individual differences in the form of the pleasantness function may be due, at least in part, to contextual effects. Subjects may come into the experimetnal task with judgmental contexts already established by experience. These can affect the location of the breakpoint in the same way it is affected by manipulations of context in the laboratory. In contrast to subjects in prior experiments, the subjects in the present study may be relatively homogeneous with respect to judgmental context, so that their breakpoints all fall within the range of concentrations tested.

\section{REFERENCES}

Amerine, M. A., Pangborn, R. M., \& Roessler, E. B. Principles of sensory evaluation of food. New York: Academic Press, 1965.

Chappelt, G. Flavour assessment of sugar solutions. Journal of Science and Agriculture, 1953, 4, 346-350.

Helson, H. Adaptation-level theory. New York: Harper \& Row, 1964.

Kocher, E. C., \& Fisher, G. L. Subjective intensity and taste preference. Perceptual and Motor Skills, 1969, 28, 735-740. 
Moskowitz, H. R. The sweetness and pleasantness of sugars. American Journal of Psychology, 1971, 84, 387-405.

Moskowitz, H. R. Sensations, measurement and pleasantness: Confessions of a latent introspectionist. In J. M. Weiffenbach (Ed.), Taste and development: The genesis of sweet preference. Bethesda, Md: U.S. Department of Health, Education \& Welfare, 1977. (a)

Moskowitz, H. R. Intensity and hedonic functions for chemosensory stimuli. In M. R. Kare \& O. Maller (Eds.), The chemical senses and nutrition. New York: Academic Press, 1977. (b)

Moskowitz, H. R., Kluter, R. A., Westerling, J., \& Jacobs, H. L. Sugar sweetness and pleasantness: Evidence for different psychological laws. Science, 1974, 184, 583-585.

Moskowitz, H. R., Kumraiah, V., Sharma, K. N., Jacobs, H. L., \& Sharma, S. D. Cross-cultural differences in simple taste preferences. Science, 1975, 190, 1217-1218.

O'Mahony, M., \& Dunn, M. Do sensitivity drifts occur for stimuli other than sodium chloride? A preliminary investigation. Perception, 1974, 3, 213-220.

Pangborn, R. M. Individual variations in affective responses to taste stimuli. Psychonomic Science, 1970, 21, 125-126.

Parducci, A. Range-frequency compromise in judgment. Psychological Monographs, 1963, 77(2, Whole No. 565).

Parducci, A. Category judgment: A range-frequency model. Psychological Review, 1965, 72, 407-418.

PArducci, A. Category judgment: A range-frequency analysis. In E. C. Carterette \& M. P. Friedman (Eds.), Handbook of .perception (Vol. 11). New York: Academic Press, 1974.
Parducci, A., Calfee, R. C., Marshall, L. M., \& Davidson, L. P. Context effects in judgment: Adaptation level as a function of the mean, midpoint, and median of the stimuli. Journal of Experimental Psychology, 1960, 60, 65-77.

Parducci, A., \& Perrett, L. P. Category rating scales: Effects of relative spacing and frequency of stimulus values. Journal of Experimental Psychology Monograph, 1971, 89, 427-452.

Poulton, E. C. The new psychophysics: Six models for magnitude estimation. Psychological Bulletin, 1968, 69, 1-19.

Sandusky, A., \& Parducci, A. Pleasantness of odors as a function of the immediate stimulus context. Psychonomic Science, $1965,3,321-322$.

Stevens, S. S. Adaptation-level vs. the relativity of judgment. American Journal of Psychology, 1958, 71, 633-647.

Ward, L. M., \& Lockniad, G. R. Response system processes in absolute judgment. Perception \& Psychophysics, 1971, 9. 73-78.

\section{NOTE}

1. Kool-Aid unsweetened soft drink mix (General Food Corporation) was used as flavoring. Each liter of beverage contained $3.5 \mathrm{~g}$ of flavoring.

(Received for publication February 27, 1979; revision accepted July $16,1979$. ) 